Pacific Journal of Mathematics

ON THE DECOMPOSITION OF INFINITELY DIVISIBLE
PROBABILITY LAWS WITHOUT NORMAL FACTOR 


\section{ON THE DECOMPOSITION OF INFINITELY DIVISIBLE PROBABILITY LAWS WITHOUT NORMAL FACTOR}

\section{ROGER CUPPENS}

In the theory of the decomposition of probability laws, the fundamental problem stated by D. A. Raikov of the characterization of the class $I_{0}$ of the infinitely divisible laws without indecomposable factors has been studied in the case of univariate laws by Yu. V. Linnik and I. V. Ostrovskiy. Lately, we have shown that nearly all these results can be extended to the case of multivariate laws. In this paper, we give a result which can be considered as an extension of a theorem of Raikov and P. Lévy and of a particular case of theorems of Linnik, and the extension of this result to the case of several variables.

If we consider the finite products of Poisson laws, i.e., the characteristic functions of the variable $t$ of the form

$$
f(t)=\exp \left\{i c t+\sum_{j=1}^{p} \lambda_{j}\left[\exp \left(i \alpha_{j} t\right)-1\right]\right\}
$$

( $c$ real, $\lambda_{j}>0, \alpha_{j}>0$ ), three general results are known, the first being owed to D. A. Raikov [9] and P. Lévy [4] and the third to Yu. V. Linnik [5, Chapter 9]:

(a) if $\alpha_{1}, \cdots, \alpha_{p}$ are rationally independent, $f$ has no indecomposable factor;

(b) if $\alpha_{1}, \cdots, \alpha_{p}$ are such that $0<a \leqq \alpha_{j} \leqq 2 a(j=1, \cdots, p), f$ has no indecomposable factor;

(c) if $\alpha_{j+1} / \alpha_{j}$ is an integer greater than $1(j=1, \cdots, p-1), f$ has no indecomposable factor.

Lately, I. V. Ostrovskiy [8] has extended the two results (a) and (b) of Raikov and Lévy to the case of a continuous spectrum, the base of his study being the

THEOREM 1. (see also [1] chapter 8). Let $f_{0}$ be the infinitely divisible characteristic function of the variable $t$ defined by

$$
f_{0}(t)=\exp \left\{i \gamma t+\int_{a}^{b}[\exp (i x t)-1] d \mu(x)\right\},
$$

where $\gamma$ is a real constant and $\mu$ is a nonnegative measure defined on the segment $[a, b](0<a<b<\infty)$. If $f_{1}$ is a factor of $f_{0}$, then

$$
f_{1}(t)=\exp \left\{i c t+\int_{a}^{b}[\exp (i x t)-1] d m(x)\right\},
$$


where $c$ is a real constant and $m$ is a measure defined on the segment $[a, b]$ which is nonnegative on $[a, 2 a[$. Moreover,

$$
S(m) \subset[a, b] \cap(\infty S(\mu)),
$$

where $S(N)$ means the support of a measure $N$ and $(\infty A)$ is defined by

$$
(1) A=A ; \quad(p) A=(p-1) A+A ; \quad(\infty A)=\bigcup_{p=1}^{\infty}(p) A
$$

(the symbol + indicates the vectorial sum of two subsets of $R$ ).

He gives also a more general result which can be stated in the following manner:

THEOREM 2. Let $f_{0}$ be the infinitely divisible characteristic function of the variable $t$ defined by

$$
f_{0}(t)=\exp \left\{i \gamma t+\int_{a}^{b}[\exp (i x t)-1] d \mu(x)+\sum_{k=1}^{\infty} \lambda_{k}\left[\exp \left(i \alpha_{k} t\right)-1\right]\right\},
$$

where $\gamma \in R, \lambda_{k} \geqq 0, \alpha_{k}>0(k=1,2, \cdots)$ and where the following conditions are satisfied:

(1) the measure $\mu$ is a nonnegative measure defined on the segment $[a, b](0<a<b<\infty)$;

(2) there exists a positive constant $K$ such that

$$
\lambda_{k}=0\left[\exp \left(-K \alpha_{k}^{2}\right)\right] \quad(k \rightarrow+\infty) ;
$$

(3) $\alpha_{1}>b$ and $\alpha_{k+1} / \alpha_{k}$ is an integer greater than $1(k=1,2, \cdots)$. If $f_{1}$ is a factor of $f_{0}$, then

$$
f_{1}(t)=\exp \left\{i c t+\int_{a}^{b}[\exp (i x t)-1] d m(x)+\sum_{k=1}^{\infty} l_{k}\left[\exp \left(i \alpha_{k} t\right)-1\right]\right\},
$$

where $c$ is a real constant and the following conditions are satisfied:

(a) $\quad 0 \leqq l_{k} \leqq \lambda_{k} \quad(k=1,2, \cdots)$;

(b) the measure $m$ is a measure defined on the segment $[a, b]$ which is nonnegative on $[a, 2 a[$ and such that

$$
m(\{b\}) \geqq 0, \quad S(m) \subset[a, b] \cap(\infty S(\mu)) .
$$

Using the Theorems 1 and 2, we give in $\S 2$, two theorems which can be considered as extensions of the results (a) and (c) stated above. Using the auxiliary results stated in $\S 3$, we extend these results to the case of several variables in the $\S 4$.

2. The case of one variable.

THEOREM 3. Let $f_{0}$ be the infinitely divisible characteristic 
function of the variable $t$ defined by

$$
f_{0}(t)=\exp \left\{i \gamma t+\sum_{j=1}^{p} \sum_{k=1}^{r_{j}} \lambda_{j, k}\left[\exp \left(i \alpha_{j, k} t\right)-1\right]\right\},
$$

where $\gamma$ is a real constant, the $\lambda_{j, k}$ are nonnegative constants and the $\alpha_{j, k}$ are positive numbers satisfying the two conditions

(a) $\alpha_{j, k+1} / \alpha_{j, k}$ is an integer greater than $1\left(k=1, \cdots, r_{j}-1 ; j=\right.$ $1, \cdots, p)$

(b) $\alpha_{1,1}, \cdots, \alpha_{p, 1}$ are rationally independent. If $f_{1}$ is a factor of $f_{0}$, then

$$
f_{1}(t)=\exp \left\{i c t+\sum_{j=1}^{p} \sum_{k=1}^{r_{j}} l_{j, k}\left[\exp \left(i \alpha_{j, k} t\right)-1\right]\right\},
$$

where $c$ is a real constant and the $l_{j, k}$ satisfy

$$
0 \leqq l_{j, k} \leqq \lambda_{j, k}
$$

Proof. Let $f_{1}$ and $f_{2}$ be the two characteristic functions such that for any real $t$

$$
f_{0}(t)=f_{1}(t) f_{2}(t) \text {. }
$$

Since $f_{0}$ is an entire characteristic function, from Raikov's theorem ([6], theorem 8.1.1), $f_{j}(j=1,2)$ is also entire and the equation (2.1) is also valid for any complex $t$. Moreover, we have the ridge property ([6], Theorem 7.1.2) which can be written, since $f_{j}$ is evidently without zeros,

$$
u_{j}(0, y)-u_{j}(x, y) \geqq 0 \quad(j=1,2)
$$

for any real $x$ and $y$ where $u_{j}$ is defined by

$$
u_{j}(x, y)=\operatorname{Re} \log f_{j}(x+i y) .
$$

From the Theorem 1 of the introduction, it follows that for any complex $t$

$$
f_{1}(t)=\exp \left\{i c t+\sum_{j=1}^{p} \sum_{k=1}^{s_{j}} l_{j, k}^{\prime}\left[\exp \left(i k \alpha_{j, 1} t\right)-1\right]\right\},
$$

where $c$ and the $l_{j, k}^{\prime}$ are real constants and where $s_{j}$ is defined by

$$
s_{j} \alpha_{j, 1} \leqq \sup _{k} \alpha_{k, r_{k}}<\left(s_{j}+1\right) \alpha_{j, 1}
$$

From (2.3), it follows by an elementary computation that

$$
u_{1}(0, y)-u_{1}(x, y)=2 \sum_{j=1}^{p} \sum_{k=1}^{s_{j}} l_{j, k}^{\prime} \sin ^{2}\left(\frac{1}{2} k \alpha_{j, 1} x\right) \exp \left(k \alpha_{j, 1} y\right) .
$$

We show now by induction that all the $l_{j, k}^{\prime}$ for $k \alpha_{j, 1} \notin\left\{\alpha_{j, k}\right\}$ are 
equal to zero and that all the $l_{j, k}^{\prime}$ for $k \alpha_{j, 1} \in\left\{\alpha_{j, k}\right\}$ are nonnegative. (It is sufficient to show that all the $l_{j, k}^{\prime}$ are nonnegative since if $l_{j, k}^{\prime}$ for $k \alpha_{j, 1} \notin\left\{\alpha_{j, k}\right\}$ is nonnegative, the corresponding term in $f_{2}$ is also nonnegative and their sum is zero).

First of all, we show that

$$
l_{j, s_{j}}^{\prime} \geqq 0 .
$$

Indeed, from Kronecker's theorem ([3], Theorem 444), it is possible to find $x=x(y)$ such that

and

$$
\begin{aligned}
\sin \left(\frac{1}{2} k \alpha_{j^{\prime}, 1} x\right)=o\left(\exp \left[-\frac{1}{2} s_{j^{\prime}} \alpha_{j^{\prime}, 1} y\right]\right) & \\
(y \rightarrow \infty) \quad j^{\prime} & \neq j, k=1, \cdots, s_{j^{\prime}}
\end{aligned}
$$

$$
\sin \left(\frac{1}{2} s_{j} \alpha_{j, 1} x\right) \geqq 1-\varepsilon .
$$

We have then from $(2.4)$

$$
u_{1}(0, y)-u_{1}(x, y)=O\left[l_{j, s j}^{\prime} \exp \left(s_{j} \alpha_{j, 1} y\right)\right]
$$

when $y \rightarrow \infty$ and (2.2) implies (2.5).

Let now $k<s_{j}$ and let $\nu$ be the smallest integer greater than $k$ such that $l_{j, \nu}^{\prime}>0$ (if such a $\nu$ does not exist, the preceding proof is still valid). From the hypothesis of induction, we can suppose that $l_{j, k^{\prime}}^{\prime}$ is zero if $k^{\prime}(>k)$ is not a multiple of $\nu$. From Kronecker's theorem, it is possible to find $x=x(y)$ and an integer $p_{j}$ such that (2.6) and

$$
x \alpha_{j, 1}-2 p_{j} \pi-\frac{2 \pi}{\nu}=o\left(\exp \left[-\frac{1}{2} s_{j} \alpha_{j, 1} y\right]\right) \quad(y \rightarrow \infty)
$$

are satisfied. We have then from (2.4)

$$
u_{1}(0, y)-u_{1}(x, y)=O\left[l_{j, k}^{\prime} \sin ^{2}\left(\frac{1}{2} k \alpha_{j, 1} x\right) \exp \left(k \alpha_{j, 1} y\right)\right]
$$

and

$$
\sin ^{2}\left(\frac{1}{2} k \alpha_{j, 1} x\right) \geqq c>0 \text {. }
$$

It follows from (2.2) that

$$
l_{j, k}^{\prime} \geqq 0
$$

and the theorem is demonstrated.

We can generalize the Theorem 3 in the following manner:

THEOREM 4. Let $f_{0}$ be the infinitely divisible characteristic function of the variable $t$ defined by

$$
f_{0}(t)=\exp \left\{i \gamma t+\sum_{j=1}^{p} \sum_{k=1}^{r_{j}} \lambda_{j, k}\left[\exp \left(i \alpha_{j, k} t\right)-1\right]+\sum_{q=1}^{\infty} \mu_{q}\left[\exp \left(i \beta_{q} t\right)-1\right]\right\},
$$


where the following conditions are satisfied

(1) $\gamma$ is a real constant;

(2) the $\lambda_{j, k}$ and the $\mu_{q}$ are nonnegative constants and there exists a positive constant $K$ such that

$$
\mu_{q}=O\left[\exp \left(-K \beta_{q}^{2}\right)\right] \quad(q \rightarrow \infty) .
$$

(3) the $\alpha_{j, k}$ and the $\beta_{q}$ are positive constants such that

(a) $\alpha_{j, k+1} / \alpha_{j, k} \quad\left(k=1, \cdots, r_{j}-1 ; j=1, \cdots, p\right)$ and $\beta_{q+1} / \beta_{q}$ $(q=1,2, \cdots)$ are integers greater than 1 ;

(b) $\alpha_{1,1}, \cdots, \alpha_{p, 1}$ and $\beta_{1}$ are rationally independent. If $f_{1}$ is $a$ factor of $f_{0}$, then

$$
f_{1}(t)=\exp \left\{i c t+\sum_{j=1}^{p} \sum_{k=1}^{r_{j}} l_{j, k}\left[\exp \left(i \alpha_{j, k} t\right)-1\right]+\sum_{q=1}^{\infty} m_{q}\left[\exp \left(i \beta_{q} t\right)-1\right]\right\},
$$

where $c$ is a real constant and the $l_{j, k}$ and the $m_{q}$ satisfy

$$
0 \leqq l_{j, k} \leqq \lambda_{j, k} ; \quad 0 \leqq m_{q} \leqq \mu_{q} \text {. }
$$

Proof. The proof is essentially the same as the preceding. Using the Theorem 2 of the introduction, we obtain the representation

$$
\begin{aligned}
f_{1}(t)= & \exp \left\{i c t+\sum_{j=1}^{p} \sum_{k=1}^{s_{j}} l_{j, k}^{\prime}\left[\exp \left(i k \alpha_{j, 1} t\right)-1\right]\right. \\
& \left.+\sum_{q=1}^{o} m_{q}^{\prime}\left[\exp \left(i q \beta_{1} t\right)-1\right]+\sum_{q=\tau}^{\infty} m_{q}\left[\exp \left(i \beta_{q} t\right)-1\right]\right\},
\end{aligned}
$$

where $c$, the $l_{j, k}^{\prime}$ and the $m_{q}^{\prime}$ are real constants, the $m_{q}$ satisfy

$$
0 \leqq m_{q} \leqq \mu_{q}
$$

and where $s_{j}, \sigma$ and $\tau$ are defined by $\left(d=\sup _{j} \alpha_{j, r}\right)$

$$
\begin{aligned}
& s_{j} \alpha_{j, 1} \leqq d<\left(s_{j}+1\right) \alpha_{j, 1} \text {, } \\
& \sigma \beta_{1} \leqq d<(\sigma+1) \beta_{1}, \\
& \beta_{\tau-1} \leqq d<\beta_{\tau} \text {. }
\end{aligned}
$$

The proof of the nonnegativity of all the $l_{j, k}^{\prime}$ and of all the $m_{q}^{\prime}(q \leqq \sigma)$ (which implies that all the $l_{j, k}^{\prime}$ for $k \alpha_{j, 1} \notin\left\{\alpha_{j, k}\right\}$ and all the $m_{q}^{\prime}$ for $q \beta_{1} \notin\left\{\beta_{q}\right\}$ are zero) is the same except that we use instead of the Theorem 444 of [3]) the other form of Kronecker's theorem (Theorem 443 of [3]) which asserts that the values of $x$ satisfying (2.6) and (2.7) (or (2.6) and (2.8)) can be taken in the form $2 \kappa \pi / \beta_{q}$ ( $\kappa$ integer).

3. Some auxiliary results. We enumerate now some results which are useful in the following section. 
Lemma 1. ([7], Corollary of the Theorem 1). Let $f$ be a function of the complex variable $z$, analytic in the half-plane $\operatorname{Re} z \geqq 0$ and satisfying the conditions
(1) $|f(z)| \leqq M_{1}|z+1|^{a}$
(2) $|f(z)| \leqq M_{2}(z+1)^{c} \exp (b z)$ for $\operatorname{Re} z=0$,
(3) $|f(z)| \leqq M_{3}|z+1|^{\circ} \exp \left[d(\operatorname{Re} z)^{2}\right]$ for $\operatorname{Re} z \geqq 0$, where $M_{1}, M_{2}, M_{3}$ are positive constants and $a, b, c(\geqq a)$ and $d$ are nonnegative constants. Then in all the half-plane $\operatorname{Re} z \geqq 0$

$$
|f(z)| \leqq M_{1}|z+1|^{a} \exp (b \operatorname{Re} z) .
$$

Lemma 2. Let $f$ be a function of the $n$ complex variables $z=$ $\left(z_{1}, \cdots, z_{n}\right)$ admitting the representation

$$
f(z)=\sum_{p_{1}=0}^{\infty} \cdots \sum_{p_{n}=0}^{\infty} d_{p_{1}, \cdots, p_{n}} \exp \left(2 \pi \sum_{j=1}^{n} \frac{p_{j} z_{j}}{T_{j}}\right),
$$

where $T_{j}>0(j=1, \cdots, n)$. In order that the constants $d_{p_{1}, \cdots, p_{n}}$ satisfy for some $K>0$ the relation

$$
d_{p_{1}, \ldots, p_{n}}=O\left(\exp \left(-K \sum_{j=1}^{n} p_{j}^{2}\right)\right) \quad\left(\sum_{j=1}^{n} p_{j} \rightarrow \infty\right),
$$

it is necessary that $f$ be an entire function satisfying

$$
\ln |f(z)|=O\left(\sum_{j=1}^{n}\left|\operatorname{Re} z_{j}\right|^{2}\right) \quad(|z| \rightarrow \infty)
$$

and sufficient that $f$ be an entire function satisfying

$$
\begin{aligned}
\ln |f(z)| & =O\left(\sum_{j=1}^{n} \mid\left[\left.\operatorname{Re} z_{j}\right|^{2}+\ln |z|\right)\right. \\
(|z| & \rightarrow \infty) \quad\left(|z|^{2}=\sum_{j=1}^{n}\left|z_{j}\right|^{2}\right) .
\end{aligned}
$$

In the case $n=1$, this lemma is a particular case of the Theorem 2 of [7]. The proof in the general case is the same as in [7] and is therefore omitted.

LEMMA 3. If the entire function $f$ of the variable $z$ satisfies for some real $K$ the condition

$$
|f(z)| \leqq \exp [K \operatorname{Re} z+O(\ln |z|)] \quad(\operatorname{Re} z \geqq 0)
$$

when $|z| \rightarrow \infty$ and admits an expansion of the form

$$
f(z)=\sum_{p=-\infty}^{+\infty} a_{p} \exp (2 \pi p z / T)
$$


where $T>0$ and the series converges uniformly in every bounded set, then

$$
a_{p}=0
$$

for

$$
p>\left[K T(2 \pi)^{-1}\right] .
$$

This lemma is a particular case of the Theorem 3 of [7].

LEMMA 4. If $\varphi$ is an entire function of the $n$ variables $z=$ $\left(z_{1}, \cdots, z_{n}\right)$ such that for any $x, y \in R^{n}$ and any $\varepsilon>0$

$$
\begin{aligned}
& u(x, y)=\operatorname{Re} \varphi(x+i y)=O[\exp (\tau+\varepsilon)(|x|+|y|)] \\
& \quad(|x|+|y| \rightarrow \infty),
\end{aligned}
$$

then $\varphi$ is a function of exponential type $\tau$ with respect to the hermitian norm.

This lemma is a particular case of the Lemma 2 of the theorem 2.5 of [1].

For the following lemma, we recall the

DEFINITION. Let $\left\{f_{n}\right\}$ be a sequence of functions belonging to a Banach space of functions. The $f_{n}$ are said topologically independent if the relation

$$
\lim _{\varepsilon \rightarrow 0}\left\|\sum_{n=1}^{\infty} \alpha_{n}(\varepsilon) f_{n}\right\|=0
$$

implies

$$
\lim _{\varepsilon \rightarrow 0} \alpha_{n}(\varepsilon)=0 \quad n=1,2, \cdots .
$$

We have then the

Lemma 5. (Lemma 1 of the Theorem 6.1 of [1]). Let $\left\{\lambda_{j}\right\} a$ sequence of real numbers such that

$$
\sum_{j=1}^{\infty} \frac{1}{\left|\lambda_{j}\right|}<+\infty
$$

Then the functions $1, z, \exp \left(\lambda_{j} z\right)(j=1,2, \cdots)$ are topologically independent in the space $C(a, b)$ of continuous functions $f$ on $[a, b]$ $(-\infty<a<b<+\infty)$ with the norm $\|f\|=\sup _{a<z<b}|f(z)|$.

Recall ([1], Chapter 4) that a function $\varphi$ of the $n$ complex variables $z=\left(z_{1}, \cdots, z_{n}\right)$ is said a ridge function if it is an entire function satisfying for any $z \in C^{n}$ the relation

$$
|\varphi(z)| \leqq \varphi(\operatorname{Re} z) \quad\left(\operatorname{Re} z=\left(\operatorname{Re} z_{1}, \cdots, \operatorname{Re} z_{n}\right)\right) .
$$


We have the

LEMMA 6. Let $\varphi_{0}$ be a ridge function of the $n$ variables $z=$ $\left(z_{1}, \cdots, z_{n}\right)$ without zeros and $\varphi_{1}$ and $\varphi_{2}$ be two ridge functions such that

$$
\varphi_{0}=\varphi_{1} \varphi_{2} \text {. }
$$

There exists a positive constant $C$ such that

$$
M\left(r ; \log \varphi_{j}\right) \leqq 6 r M\left(r+1 ; \log \varphi_{0}\right)+C r(r+1) \quad(j=1,2),
$$

where

$$
M(r ; f)=\sup _{|z|=r}|f(z)|
$$

Proof. Let

$$
\psi_{j}(z)=\log \left[\varphi_{j}(z)\right], \quad \operatorname{Re} \psi_{j}(x+i y)=u_{j}(x, y)
$$

for any $x, y \in R^{n}(j=0,1,2)$. Since $\varphi_{1}$ and $\varphi_{0} / \varphi_{1}$ are ridge functions without zeros, we have

$$
0 \leqq u_{1}(x, 0)-u_{1}(x, y) \leqq u_{0}(x, 0)-u_{0}(x, y) \leqq 2 M\left(r ; \psi_{0}\right)
$$

for $|x+i y| \leqq r$.

We estimate now $\left|u_{1}(x, 0)\right|$. For that, we use the existence for any ridge function $\varphi$ of a positive constant $C_{\varphi}$ such that

$$
\log \varphi(x) \geqq-C_{\varphi}|x|
$$

for any $x \in R^{n}$. Indeed, since $\log \varphi(\lambda \theta)$ is for any direction $\theta$ of $R^{n}$ a convex function of $\lambda$, we have

$$
\log \varphi(\lambda \theta) \geqq \log \varphi(0)+\lambda(\alpha \cdot \theta),
$$

where $\alpha=\left(\alpha_{1}, \cdots, \alpha_{n}\right), \alpha_{j} \varphi(0)=\left\{\partial \varphi(0) / \partial z_{j}\right.$ and where $(\alpha \cdot \theta)$ indicates. the scalar product of the vectors $\alpha$ and $\theta$. The relation (3.2) is an. immediate consequence of (3.3).

From (3.2), we have

$$
\begin{aligned}
& u_{1}(x, 0)=\psi_{1}(x) \geqq-C_{1}|x|, \\
& u_{1}(x, 0)=\psi_{0}(x)-\psi_{2}(x) \leqq \psi_{0}(x)+C_{2}|x| .
\end{aligned}
$$

From (3.4) and (3.5), it follows

$$
\left|u_{1}(x, 0)\right| \leqq M\left(r ; \psi_{0}\right)+C r \quad(|x| \leqq r)
$$

for some positive constant $C$ and from (3.1)

$$
\left|u_{1}(x, y)\right| \leqq 3 M\left(r ; \psi_{0}\right)+C r(|x+i y| \leqq r) .
$$

Let now $g_{\theta}$ the function of the complex variable $\lambda$ defined by 


$$
g_{\theta}(\lambda)=\psi_{1}(\lambda \theta)
$$

for some direction $\theta$ of $R^{n}$. Then

$$
v_{\theta}(\mu, \nu)=\operatorname{Re} g_{\theta}(\mu+i \nu)=u_{1}(\mu \theta+i \nu \theta)
$$

for any real $\mu$ and $\nu$. We have then $(\lambda=\mu+i \nu)$

$$
g_{\theta}^{\prime}(\lambda)=\frac{1}{\pi} \int_{0}^{2 \pi} v_{\theta}(\mu+\cos \alpha, \nu+\sin \alpha) e^{-i \alpha} d \alpha ;
$$

so that

$$
\begin{aligned}
\left|g_{\theta}^{\prime}(\lambda)\right| & \leqq 2 \sup _{0 \leqq \alpha \leqq 2 \pi}\left|v_{\theta}(\mu+\cos \alpha, \nu+\sin \alpha)\right| \\
& \leqq 6 M\left(r+1 ; \log \varphi_{0}\right)+C(r+1)
\end{aligned}
$$

$(|\lambda| \leqq r)$ for some positive constant $C$. Since

$$
g_{\theta}(\lambda)=\int_{0}^{\mu} g_{\theta}^{\prime}(\xi) d \xi+i \int_{0}^{\nu} g_{\theta}^{\prime}(\mu+i \nu) d \eta
$$

and since $\theta$ is arbitrary, the lemma is a consequence of (3.6).

LEMma 7. (Lemma of the Theorem 5 of [2]). If $f$ is an entire characteristic function of the two variables $t_{1}$ and $t_{2}$ and $t_{2}^{0}$ a real constant, the function $f_{t_{2}^{0}}$ defined by

$$
f_{t_{2}^{0}}\left(t_{1}\right)=\frac{f\left(t_{1}, i t_{2}^{0}\right)}{f\left(0, i t_{2}^{0}\right)}
$$

is an entire characteristic function.

4. The case of several variables. First of all, we consider the case of functions of two variables.

THEOREM 5. Let $f_{0}$ be the infinitely divisible characteristic function of the two variables $t=\left(t_{1}, t_{2}\right)$ defined by

$$
\begin{aligned}
f_{0}(t)= & \exp \left\{i \pi(t)+\sum_{j=1}^{\infty}\left(\lambda_{j}\left[\exp \left(i \alpha_{j} t_{1}\right)-1\right]\right.\right. \\
& \left.\left.+\mu_{j}\left[\exp \left(i \beta_{j} t_{2}\right)-1\right]+\nu_{j}\left[\exp \left(i \alpha_{j} t_{1}+i \beta_{j} t_{2}\right)-1\right]\right)\right\},
\end{aligned}
$$

where the following conditions are satisfied

(1) $\pi$ is an homogeneous polynomial of degree one with real coefficients;

(2) $\lambda_{j}, \mu_{j}, \nu_{j}$ are nonnegative constants and there exists a positive constant $K$ such that 


$$
\begin{aligned}
& \lambda_{j}=O\left[\exp \left(-K \alpha_{j}^{2}\right)\right] ; \quad \mu_{j}=O\left[\exp \left(-K \beta_{j}^{2}\right)\right], \\
& \nu_{j}=O\left[\exp \left(-K\left(\alpha_{j}^{2}+\beta_{j}^{2}\right)\right)\right] \quad(j \rightarrow+\infty) ;
\end{aligned}
$$

(3) the $\alpha_{j}$ are positive constants satisfying the three conditions

(a) there exists $q_{1}$ such that $\alpha_{j+1} / \alpha_{j}$ is an integer greater than 1 for $j \geqq q_{1}$;

(b) the set $\left\{\alpha_{j} ; j<q_{1}\right\}$ can be decomposed in $p$ sets $\left\{\alpha_{j, k}\right\}$ $\left(j=1, \cdots, p ; k=1, \cdots, r_{j} ; \sum_{j=1}^{p} r_{j}=q_{1}-1\right)$ such that $\alpha_{j, k+1} / \alpha_{j, k}\left(k=1, \cdots, r_{j}-1 ; j=1, \cdots, p\right)$ is an integer greater than 1 and $\alpha_{1,1}, \cdots, \alpha_{p, 1}$ are rationally independent;

(c) either $\alpha_{q_{1}}$ is a multiple of one of the $\alpha_{j, r}$ or $\alpha_{1,1}, \cdots, \alpha_{p, 1}$ and $\alpha_{q_{1}}$ are rationally independent;

(4) the $\beta_{j}$ are positive constants having the same property. If $f_{1}$ is a factor of $f_{0}$, then

$$
\begin{aligned}
f_{1}(t)= & \exp \left\{i P(t)+\sum_{j=1}^{\infty}\left(l_{j}\left[\exp \left(i \alpha_{j} t_{1}\right)-1\right]\right.\right. \\
& \left.\left.+m_{j}\left[\exp \left(i \beta_{j} t_{2}\right)-1\right]+n_{j}\left[\exp \left(i \alpha_{j} t_{1}+i \beta_{j} t_{2}\right)-1\right]\right)\right\}
\end{aligned}
$$

where $P$ is an homogeneous polynomial of degree one with real coefficients and where $l_{j}, m_{j}, n_{j}$ are constants satisfying the conditions

$$
0 \leqq l_{j} \leqq \lambda_{j} ; \quad 0 \leqq m_{j} \leqq \mu_{j}, \quad 0 \leqq n_{j} \leqq \nu_{j} .
$$

Proof. Let $f_{1}$ and $f_{2}$ be the two characteristic functions such that for any real $t_{1}$ and $t_{2}$

$$
f_{0}\left(t_{1}, t_{2}\right)=f_{1}\left(t_{1}, t_{2}\right) f_{2}\left(t_{1}, t_{2}\right) .
$$

Since $f_{0}$ is an entire characteristic function, from Raikov's theorem ([1], Theorem 2.3), $f_{j}$ is also entire $(j=1,2)$ and the equation (4.1) is also valid for any complex $t_{1}$ and $t_{2}$, Letting

$$
\begin{aligned}
\varphi_{j}(z) & =f_{j}(-i z), \\
u_{j}(x, y) & =\operatorname{Re} \log \varphi_{j}(x+i y),
\end{aligned}
$$

$(j=0,1,2)$ for any $x, y \in R^{2}$, since $\varphi_{j}$ is a ridge function ([1], Corollary 1 of the Theorem 2.1), we have

$$
0 \leqq u_{1}(x, 0)-u_{1}(x, y) \leqq u_{0}(x, 0)-u_{0}(x, y)
$$

for any $x, y \in R^{2}$.

If we fix $z_{2}$ real, using the Lemma 7 and the Theorem 4 , we have

$$
\log \varphi_{1}(z)=a+b z_{1}+\sum_{j=1}^{\infty} c_{j} \exp \left(\alpha_{j} z_{1}\right),
$$

where $a, b, c_{j}$ are functions of $z_{2}$, real for $z_{2}$ real and satisfying 


$$
0 \leqq c_{j}\left(z_{2}\right) \leqq \lambda_{j}+\nu_{j} \exp \left(\beta_{j} z_{2}\right)
$$

If we fix $z_{1}$ real, we have

$$
\log \varphi_{1}(z)=r+s z_{2}+\sum_{j=1}^{\infty} t_{j} \exp \left(\beta_{j} z_{2}\right),
$$

where $r, s, t_{j}$ are functions of $z_{1}$, real for $z_{1}$ real and satisfying

$$
0 \leqq t_{j}\left(z_{1}\right) \leqq \mu_{j}+\nu_{j} \exp \left(\alpha_{j} z_{1}\right) \text {. }
$$

From (4.3) and (4.5), we obtain the equation for any real $z_{1}$ and $z_{2}$

$$
a+b z_{1}+\sum_{j=1}^{\infty} c_{j} \exp \left(\alpha_{j} z_{1}\right)=r+s z_{2}+\sum_{k=1}^{\infty} t_{2} \exp \left(\beta_{k} z_{2}\right)
$$

which can be solved by using the Lemma 5 (for the details, see the proof of the Theorem 6.1 of [1|). We obtain for any $z_{1}$ and $z_{2}$ complex the representation

$$
\begin{aligned}
\log \varphi_{1}(z) & =c+P(z)+d z_{1} z_{2}+\sum_{j=1}^{\infty}\left[\rho_{j} z_{2} \exp \left(\alpha_{j} z_{1}\right)+\sigma_{j} z_{1} \exp \left(\beta_{j} z_{2}\right)\right] \\
& +\sum_{j=0}^{\infty} \sum_{k=0}^{\infty} n_{j, k} \exp \left(\alpha_{j} z_{1}+\beta_{k} z_{2}\right)
\end{aligned}
$$

where all the constants and the coefficients of the homogeneous polynomial $P$ of degree one are real (with the convention $\alpha_{0}=\beta_{0}=n_{0,0}=0$ ). By an elementary computation, we obtain

$$
\begin{aligned}
u_{1}(x, 0)-u_{1}(x, y)= & d y_{1} y_{2}+\sum_{j=1}^{\infty}\left[2 \rho_{j} x_{2} \exp \left(\alpha_{j} x_{1}\right) \sin ^{2}\left(\frac{1}{2} \alpha_{j} y_{1}\right)\right. \\
& +2 \sigma_{j} x_{1} \exp \left(\beta_{j} x_{2}\right) \sin ^{2}\left(\frac{1}{2} \beta_{j} y_{2}\right) \\
& +\rho_{j} y_{2} \exp \left(\alpha_{j} x_{1}\right) \sin \left(\alpha_{j} y_{1}\right) \\
& \left.+\sigma_{j} y_{1} \exp \left(\beta_{j} x_{2}\right) \sin \left(\beta_{j} y_{2}\right)\right] \\
& +2 \sum_{j=0}^{\infty} \sum_{k=0}^{\infty} n_{j, k} \exp \left(\alpha_{j} x_{1}+\beta_{k} x_{2}\right) \sin ^{2}\left(\frac{\alpha_{j} y_{1}+\beta_{k} y_{2}}{2}\right) .
\end{aligned}
$$

Letting $\left|y_{1}\right| \rightarrow \infty$, we obtain from (4.2) and (4.8)

$$
d y_{2}+\sum_{j=1}^{\infty} \sigma_{j} \exp \left(\beta_{j} x^{2}\right) \sin \left(\beta_{j} y_{2}\right)=0 .
$$

Since the expression in the left member is the imaginary part of

$$
d z_{2}+\sum_{j=1}^{\infty} \sigma_{j} \exp \left(\beta_{j} z_{2}\right)
$$

we deduce from the Lemmas 4 and 3 that

$$
d=\sigma_{j}=0 \text {. }
$$


In the same manner, letting $\left|y_{2}\right| \rightarrow \infty$, we obtain

$$
\rho_{j}=0 \text {. }
$$

From the Lemma 5 and (4.4), it follows that for any real $x_{2}$

$$
0 \leqq \sum_{k=0}^{\infty} n_{j, k} \exp \left(\beta_{k} x_{2}\right) \leqq \lambda_{j}+\nu_{j} \exp \left(\beta_{j} x_{2}\right) \text {. }
$$

On the other hand, $\log \varphi_{0}$ satisfies

$$
\log \varphi_{0}(z)=O\left[|z|\left(1+\exp \left(N|\operatorname{Re} z|^{2}\right)\right)\right] \quad(|z| \rightarrow \infty)
$$

for some $N>0$. It follows from the lemma 6 that

$$
\log \varphi_{1}(z)=O\left[|z|^{2}\left(1+\exp \left(N|\operatorname{Re} z|^{2}\right)\right)\right] \quad(|z| \rightarrow \infty)
$$

and from the sufficient part of the Lemma 2 applied to

$$
\sum_{j=q_{1}}^{\infty} \sum_{k=q_{2}}^{\infty} n_{j, k} \exp \left(\alpha_{j} z_{1}+\beta_{k} z_{2}\right)
$$

(the constant $q_{1}$ is defined in the statement of the theorem and the constant $q_{2}$ is the analogous for the $\beta_{k}$ ), we have for some $\kappa^{\prime}>0$

$$
n_{j, k}=O\left[\exp \left(-\kappa^{\prime}\left(j^{2}+k^{2}\right)\right)\right] \quad(|j|+|k| \rightarrow \infty),
$$

that implies from the necessary part of the Lemma 2

$$
\sum_{k=0}^{\infty} n_{j, k} \exp \left(\beta_{k} z_{2}\right)=O\left[\exp \left(N^{\prime}\left|\operatorname{Re} z_{2}\right|^{2}\right)\right] \quad\left(\left|z_{2}\right| \rightarrow \infty\right)
$$

for any complex $z_{2}$ and some $N^{\prime}>0$. Using the Lemma 1, we obtain

$$
\sum_{k=0}^{\infty} n_{j, k} \exp \left(\beta_{k} z_{2}\right)=O\left[\exp \left(\beta_{j} z_{2}\right)\right] \quad\left(\left|z_{2}\right| \rightarrow \infty\right)
$$

in $\left\{\operatorname{Re} z_{2} \geqq 0\right\}$, that implies from the Lemma 3

$$
n_{j, k}=0
$$

for all the $k$ such that $\beta_{k}>\beta_{j}$ and from (4.9)

$$
n_{j, j} \geqq 0, \quad n_{j, 0} \geqq 0 .
$$

In the same manner, from (4.6), we obtain

$$
n_{j, k}=0
$$

for all the $j$ such that $\alpha_{j}>\alpha_{k}$ and

$$
n_{0, j} \geqq 0 \text {. }
$$

In particular, we have $\left(q=\sup \left(q_{1}, q_{2}\right)\right)$ 


$$
n_{j, k}=0
$$

if $(j, k) \notin\{(j, j),(0, j),(j, 0)\}$ and either $j \geqq q$ or $k \geqq q$.

(4.7) becomes (with $l_{j}=n_{j, 0}, m_{j}=n_{0, j}, n_{j}=n_{j, j}$ if $j \geqq q$ ).

$\log \varphi_{1}(z)=c+P(z)$

$$
\begin{aligned}
& +\sum_{j=q}^{\infty}\left[l_{j} \exp \left(\alpha_{j} z_{1}\right)+m_{j} \exp \left(\beta_{j} z_{2}\right)+n_{j} \exp \left(\alpha_{j} z_{1}+\beta_{j} z_{2}\right)\right] \\
& +\sum_{j=0}^{q-1} \sum_{k=0}^{q-1} n_{j, k} \exp \left(\alpha_{j} z_{1}+\beta_{k} z_{2}\right)
\end{aligned}
$$

and (4.8) becomes

$$
\begin{aligned}
\frac{1}{2}\left[u_{1}(x, 0)\right. & \left.-u_{1}(x, y)\right] \\
= & \sum_{j=q}^{\infty}\left[l_{j} \exp \left(\alpha_{j} x_{1}\right) \sin ^{2}\left(\frac{1}{2} \alpha_{j} y_{1}\right)+m_{j} \exp \left(\beta_{j} x_{2}\right) \sin ^{2}\left(\frac{1}{2} \beta_{j} y_{2}\right)\right. \\
& \left.+n_{j} \exp \left(\alpha_{j} x_{1}+\beta_{j} x_{2}\right) \sin ^{2}\left(\frac{\alpha_{j} y_{1}+\beta_{j} y_{2}}{2}\right)\right] \\
& +\sum_{j=0}^{q-1} \sum_{k=0}^{q-1} n_{j, k} \exp \left(\alpha_{j} x_{1}+\beta_{k} x_{2}\right) \sin ^{2}\left(\frac{\alpha_{j} y_{1}+\beta_{k} y_{2}}{2}\right) .
\end{aligned}
$$

We show now by induction that all the $n_{j, k}(i \leqq q-1, k \leqq q-1)$ are nonnegative (that implies $n_{j, k}=0$ if $\left.(j, k) \notin\{(j, j),(j, 0),(0, j)\}\right)$. We show that this result is true for $j=j_{0}$ such that $\alpha_{j_{0}}=\sup _{j=1 \cdots, q-1} \alpha_{j}$. We put $y_{1}=2 \pi / \alpha_{q}$ and choose $y_{2}$ from Kronecker's theorem (Theorem 443 of [3]) such that

$$
y_{2}=\frac{2 \kappa^{\prime} \pi}{\beta_{l^{\prime}}}
$$

( $\kappa^{\prime}$ integer) where $\beta_{l^{\prime}}$ is the smallest number greater than $\beta_{k}$ such that $\beta_{q} / \beta_{l}$, is integer;

$$
\sin \left(\frac{\alpha_{j_{0}} y_{1}+\beta_{k^{\prime}} y_{2}}{2}\right)=o\left[\exp \left(\frac{1}{2} \beta_{k}, x_{2}\right)\right] \quad\left(x_{2} \rightarrow \infty\right)
$$

for all the $k^{\prime}$ such that $\beta_{k^{\prime}} \geqq \beta_{k}$;

$$
\sin \left(\frac{\alpha_{j_{0}} y_{1}+\beta_{k} y_{2}}{2}\right) \geqq C>0 \text {. }
$$

Then if $x_{2}$ is chosen great enough, we obtain from (4.11)

$$
u_{1}(x, 0)-u_{1}(x, y)=O\left[n_{j_{0}, k} \exp \left(\alpha_{j_{0}} x_{1}+\beta_{k} x_{2}\right) \sin ^{2}\left(\frac{\alpha_{j 0} y_{1}+\beta_{k} y_{2}}{2}\right)\right]
$$

$\left(x_{1} \rightarrow \infty\right)$, that implies with $(4.2)$

$$
n_{j_{0} k} \geqq 0 \text {. }
$$


Let now $(j, k)$ arbitrary. We can suppose that

$$
n_{j^{\prime}, k^{\prime}} \geqq 0 \quad \text { if } \quad\left(j^{\prime}, k^{\prime}\right) \in\left\{\left(j^{\prime}, j^{\prime}\right),\left(j^{\prime}, 0\right),\left(0, j^{\prime}\right)\right\}
$$

and

$$
n_{j^{\prime}, k^{\prime}}=0 \quad \text { if } \quad\left(j^{\prime}, k^{\prime}\right) \notin\left\{\left(j^{\prime}, j^{\prime}\right),\left(j^{\prime}, 0\right),\left(0, j^{\prime}\right)\right\}
$$

if either $\alpha_{j^{\prime}}>\alpha_{j}$ or $j^{\prime}=j, \beta_{k^{\prime}}>\beta_{k}$. Then we choose $y_{1}$ from Kronecker's theorem such that

$$
y_{1}=\frac{2 \kappa \pi}{\alpha_{l}}
$$

( $\kappa$ integer) where $\alpha_{l}$ is the smallest integer greater than $\alpha_{j}$ such that $\alpha_{q} / \alpha_{l}$ is integer;

$$
\sin \left(\frac{1}{2} \alpha_{j}, y_{1}\right)=o\left[\exp \left(-\frac{1}{2} \alpha_{j}, x_{1}\right)\right] \quad\left(x_{1} \rightarrow \infty\right)
$$

for all $j^{\prime}$ such that $\alpha_{j^{\prime}}>\alpha_{j}$;

$$
\left|\sin \left(\frac{1}{2} \alpha_{j} y_{1}\right)\right| \geqq c>0 \text {. }
$$

We choose now $y_{2}$ such that, from Kronecker's theorem

$$
y_{2}=\frac{2 \kappa^{\prime} \pi}{\beta_{l^{\prime}}}
$$

( $\kappa^{\prime}$ integer) where $\beta_{l^{\prime}}$ is the smallest integer greater than $\beta_{k}$ such that $\beta_{q} / \beta_{l}$, is integer;

(ß) $\quad \sin \left(\frac{\alpha_{j}, y_{1}+\beta_{j}, y_{2}}{2}\right)=o\left[\exp \left(-\frac{1}{2} \alpha_{j}, x_{1}\right)\right] \quad\left(x_{1} \rightarrow \infty\right)$ for all $j^{\prime}$ such that $\alpha_{j^{\prime}}>\alpha_{j}$;

(y) $\quad \sin \left(\frac{\alpha_{j} y_{1}+\beta_{j} y_{2}}{2}\right)=o\left[\exp \left(-\frac{1}{2} \alpha_{j} x_{1}\right)\right] \quad\left(x_{1} \rightarrow \infty\right)$

if $\beta_{j}>\beta_{k}$ (otherwise, this condition is superfluous);

$$
\left|\sin \left(\frac{\alpha_{j} y_{1}+\beta_{k} y_{2}}{2}\right)\right| \geqq C^{\prime} \geqq 0 \text {. }
$$

We have then, from (4.11), if $x_{2}$ is chosen great enough,

$$
u_{1}(x, 0)-u_{1}(x, y)=O\left[n_{j, k} \exp \left(\alpha_{j} x_{1}+\beta_{k} x_{2}\right) \sin ^{2}\left(\frac{\alpha_{j} y_{1}+\beta_{k} y_{2}}{2}\right)\right]
$$

$\left(x_{1} \rightarrow \infty\right)$, that implies

$$
n_{j, k} \geqq 0,
$$


and the theorem is demonstrated, the value of $c$ in $(4.10)$ being determined by the condition $\log \varphi_{1}(0)=0$.

From this theorem, we deduce easily by the method of the Chapters 5 and 6 of [1] the

THEOREM 6. Let $f_{0}$ be the infinitely divisible characteristic function of the $n$ variables $t=\left(t_{1}, \cdots, t_{n}\right)$ defined by

$$
f_{0}(t)=\exp \left\{i \pi(t)+\sum_{j=1}^{\infty} \sum_{\varepsilon} \lambda_{j, s}\left[\exp \left(i \sum_{k=1}^{n} \varepsilon_{k} \alpha_{j, k} t_{k}\right)-1\right]\right.
$$

where the following conditions are satisfied:

(1) $\pi$ is an homogeneous polynomial of degree one with real coefficients;

(2) $\varepsilon_{k}=0$ or 1 and $\sum_{\varepsilon}$ indicates the summation on the $2^{n}-1$ values of $\varepsilon=\left(\varepsilon_{1}, \cdots, \varepsilon_{n}\right)$ different from $(0, \cdots, 0)$;

(3) $\lambda_{j, \varepsilon}$ are nonnegative constants and there exists a positive constant $K$ such that

$$
\lambda_{j, \varepsilon}=O\left[\exp \left(-K \sum_{k=1}^{n} \varepsilon_{k} \alpha_{j, k}^{2}\right)\right] \quad(j \rightarrow+\infty) ;
$$

(4) $\left\{\alpha_{j, k}\right\}$ is, for $k=1, \cdots, n$, a sequence of positive numbers satisfying the condition (3) of the Theorem 5.

If $f_{1}$ is a factor of $f_{0}$, then

$$
f_{1}(t)=\exp \left\{i P(t)+\sum_{j=1}^{\infty} \sum_{\varepsilon} l_{j, s}\left[\exp \left(i \sum_{k=1}^{n} \varepsilon_{k} \alpha_{j, k} t_{k}\right)-1\right]\right\},
$$

where $P$ is an homogeneous polynomial of degree one with real coefficients and $l_{j, \varepsilon}$ are constants satisfying the conditions

$$
0 \leqq l_{j, \varepsilon} \leqq \lambda_{j, \varepsilon} .
$$

With the same method, we can deduce from the Theorem $1^{\prime}$ of Ostrovskiy [8] the

THEOREM 7. Let $f_{0}$ be the infinitely divisible characteristic function of the $n$ variables $t=\left(t_{1}, \cdots, t_{n}\right)$ defined by

$$
f_{0}(t)=\exp \left\{i \pi(t)+\sum_{j=1}^{\infty} \sum_{\varepsilon} \lambda_{j, \varepsilon}\left[\exp \left(i \sum_{k=1}^{n} \varepsilon_{k} \alpha_{j, k} t_{k}\right)-1\right]\right.
$$

where, beyond the conditions (1), (2), (3) of the preceding theorem, the following condition is satisfied:

(4') $\left\{\alpha_{j, k}\right\}$ is for $k=1, \cdots, n$ a sequence of increasing positive numbers such that

(a) there exists $q_{k}$ such that $\alpha_{j+1, k} / \alpha_{j, k}\left(j \geqq q_{k}\right)$ is an integer 
greater than 1;

(b) there exists a positive constant $a_{k}$ such that $a_{k} \leqq \lambda_{j, k} \leqq 2 a_{k}$ $\left(j<q_{k}\right)$.

If $f_{1}$ is a factor of $f_{0}$, then

$$
f_{1}(t)=\exp \left\{i P(t)+\sum_{j=1}^{\infty} \sum_{\varepsilon} l_{j, \varepsilon}\left[\exp \left(i \sum_{k=1}^{n} \varepsilon_{k} \alpha_{j, k} t_{k}\right)-1\right]\right\},
$$

where $P$ is an homogeneous polynomial of degree one with real coefficients and $l_{j, \varepsilon}$ are constants satisfying the conditions

$$
0 \leqq l_{j, \varepsilon} \leqq \lambda_{j, \varepsilon}
$$

\section{REFERENCES}

1. R. Cuppens, Dźcomposition des fonctions caractéristiques des vecteurs aléatoires, Publ. Inst. Statist. Univ. Paris, 16 (1967), 63-153.

2. - On finite products of Poisson-type characteristic functions of several variables (to appear in Ann. Math. Stat.).

3. G. H. Hardy and E. M. Wright, An introduction to the theory of numbers, 3rd ed Clarendon Press, Oxford, 1954.

4. P. Lévy, L'arithmétique des lois de probabilité et les produits finis de lois de Poisson, Colloque de Genève, 1938, no. 3, pp. 25-59; Actualités Sci. Ind. no. 736, Hermann, éd. 5. Yu. V. Linnik, Decomposition of probability laws (in Russian), Izdat. Leningrad Univ., Leningrad, 1960; (English translation) Oliver and Boyd, Edinburgh and London, 1964.

6. E. Lukacs, Characteristic functions, Griffin's Statistical Monographs and Courses, no. 5, Charles Griffin and Co., Ltd., London, 1960.

7. I. V. Ostrovskiy, Some theorems on the decomposition of probability laws (in Russian), Trudy Matem. Inst. Steklova 79 (1965), 198-235.

8. - On the decomposition of infinitely divisible laws without Gaussian_factor (in Russian), Zap. Mehan. Matem. Fak i Karkov. Obsc. 32 (1966), 51-72.

9. D. A. Raikov, On the decomposition of Gauss and Poisson laws (in Russian), Izv. Akad. Nauk SSSR, ser. matem. 2 (1938), 91-124.

Received December 20, 1967. This work was supported by the National Science Foundation, under grant NSF-GP-6175.

The Catholic University of America Faculté des Sciences, Montpellier 


\section{PACIFIC JOURNAL OF MATHEMATICS}

\section{EDITORS}

H. ROYDEN

Stanford University

Stanford, California

R. R Phelps

University of Washington

Seattle, Washington 98105
J. DUGUNDJI

Department of Mathematics

University of Southern California

Los Angeles, California 90007

RICHARD ARENS

University of California

Los Angeles, California 90024

\section{ASSOCIATE EDITORS}
E. F. BECKENBACH
B. H. NeumanN
F. WOLF
K. YosidA

\section{SUPPORTING INSTITUTIONS}

UNIVERSITY OF BRITISH COLUMBIA

CALIFORNIA INSTITUTE OF TECHNOLOGY

UNIVERSITY OF CALIFORNIA

MONTANA STATE UNIVERSITY

UNIVERSITY OF NEVADA

NEW MEXICO STATE UNIVERSITY

OREGON STATE UNIVERSITY

UNIVERSITY OF OREGON

OSAKA UNIVERSITY

UNIVERSITY OF SOUTHERN CALIFORNIA
STANFORD UNIVERSITY

UNIVERSITY OF TOKYO

UNIVERSITY OF UTAH

WASHINGTON STATE UNIVERSITY

UNIVERSITY OF WASHINGTON

AMERICAN MATHEMATICAL SOCIETY CHEVRON RESEARCH CORPORATION TRW SYSTEMS

NAVAL WEAPONS CENTER 


\section{Pacific Journal of Mathematics

Vol. 28, No. $1 \quad$ March, 1969

Patrick Robert Ahern, On the geometry of the unit ball in the space of real annihilating measures .............................. 1

Kirby Alan Baker, Equational classes of modular lattices ............. 9

E. F. Beckenbach and Gerald Andrew Hutchison, Meromorphic minimal surfaces ......................................... 17

Tae Ho Choe, Intrinsic topologies in a topological lattice ..............

John Bligh Conway, A theorem on sequential convergence of measures and

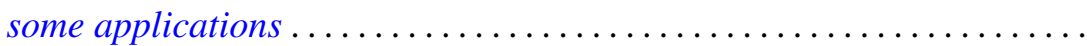

Roger Cuppens, On the decomposition of infinitely divisible probability laws without normal factor.

Lynn Harry Erbe, Nonoscillatory solutions of second order nonlinear

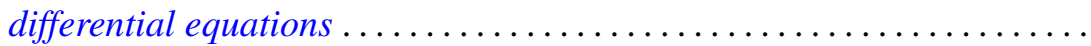

Burton I. Fein, The Schur index for projective representations of finite

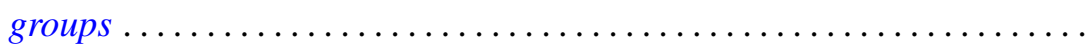

Stanley P. Gudder, A note on proposition observables............... 101

Kenneth Kapp, On Croisot's theory of decompositions ............... 105

Robert P. Kaufman, Gap series and an example to Malliavin's theorem . . . 117

E. J. McShane, Robert Breckenridge Warfield, Jr. and V. M. Warfield,

Invariant extensions of linear functionals, with applications to measures and stochastic processes ................

Marvin Victor Mielke, Rearrangement of spherical modifications ...

Akio Osada, On unicity of capacity functions ..............

Donald Steven Passman, Some 5/2 transitive permutation groups ...

Harold L. Peterson, Jr., Regular and irregular measures on groups and dyadic spaces...

Habib Salehi, On interpolation of $q$-variate stationary stochastic processes...

Michael Samuel Skaff, Vector valued Orlicz spaces generalized

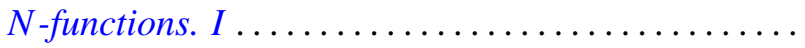

A. J. Ward, On $H$-equivalence of uniformities. II...........

Thomas Paul Whaley, Algebras satisfying the descending chain condition for subalgebras...

G. K. White, On subgroups of fixed index

Martin Michael Zuckerman, A unifying condition for implications among the axioms of choice for finite sets ................. 